TRANSACTIONS OF THE

AMERICAN MATHEMATICAL SOCIETY

Volume 358, Number 12, December 2006, Pages 5271-5291

S 0002-9947(06)04230-9

Article electronically published on July 21, 2006

\title{
LOW-PASS FILTERS AND REPRESENTATIONS OF THE BAUMSLAG SOLITAR GROUP
}

\author{
DORIN ERVIN DUTKAY
}

AbStract. We analyze representations of the Baumslag Solitar group

$$
B S(1, N)=\left\langle u, t \mid u t u^{-1}=t^{N}\right\rangle
$$

that admit wavelets and show how such representations can be constructed from a given low-pass filter. We describe the direct integral decomposition for some examples and derive from it a general criterion for the existence of solutions for scaling equations. As another application, we construct a Fourier transform for some Hausdorff measures.

\section{INTRODUCTION AND NOTATION}

Wavelet theory was developed initially for $L^{2}(\mathbb{R})$ to construct orthonormal bases that have good localization properties (see Dau92 for more details). In $L^{2}(\mathbb{R})$ we have two operators: the translation operator

$$
T f(x)=f(x-1) \quad\left(x \in \mathbb{R}, f \in L^{2}(\mathbb{R})\right)
$$

and the dilation operator

$$
U f(x)=\frac{1}{\sqrt{N}} f\left(\frac{x}{N}\right) \quad\left(x \in \mathbb{R}, f \in L^{2}(\mathbb{R})\right),
$$

where $N \geq 2$ is an integer called the scale.

A wavelet is a finite set $\left\{\psi_{1}, \ldots, \psi_{p}\right\}$ of functions in $L^{2}(\mathbb{R})$ such that

$$
\left\{U^{j} T^{k} \psi_{i} \mid j, k \in \mathbb{Z}, i \in\{1, \ldots, p\}\right\}
$$

is an orthonormal basis for $L^{2}(\mathbb{R})$.

The operators satisfy the commutation relation $U T U^{-1}=T^{N}$, hence we are dealing with a representation of the Baumslag Solitar group $B S(1, N)$. Here is the definition and some elementary facts:

The Baumslag Solitar group $B S(1, N)$ is the group with two generators $u$ and $t$ and one relation

$$
u t u^{-1}=t^{N}
$$

Received by the editors July 21, 2004.

2000 Mathematics Subject Classification. Primary 42C40, 28A78, 46L45, 28D05, 22D25.

Key words and phrases. Wavelet, representation, low-pass filter, scaling function, Baumslag Solitar group, solenoid, decomposition, ergodic action, fractal.

This work was supported in part by NSF grant DMS0457491. 
It can also be described as the semidirect product of the group of $N$-adic numbers $\mathbb{Z}[1 / N]$, with $\mathbb{Z}$, the action of $\mathbb{Z}$ on $\mathbb{Z}[1 / N]$ being given by multiplication by $N$ :

$$
\alpha_{i}\left(\frac{k}{N^{p}}\right)=N^{i} \frac{k}{N^{p}} \quad\left(i \in \mathbb{Z}, \frac{k}{N^{p}} \in \mathbb{Z}[1 / N]\right) .
$$

The elements in $\mathbb{Z}[1 / N]$ of the form $k / N^{p}$ correspond to $u^{p} t^{k} u^{-p}$ which we denote by $t_{k / N^{p}}$, and the elements in $j \in \mathbb{Z}$, correspond to $u^{j}$. Each element in the group can be written uniquely as $u^{j} t_{d}$ with $j \in \mathbb{Z}$ and $d \in \mathbb{Z}[1 / N]$. The multiplication rule is

$$
\left(u^{j} t_{d}\right)\left(u^{j^{\prime}} t_{d^{\prime}}\right)=u^{j+j^{\prime}} t_{N^{-j^{\prime}} d+d^{\prime}}
$$

The Baumslag Solitar group $B S(1, N)$ can also be regarded as an $a x+b$-group with $a$ an integer power of $N$ and $b N$-adic.

The action $\alpha$ is given by conjugation by $u$ :

$$
\alpha_{i}\left(t_{d}\right)=u^{i} t_{d} u^{-i} \quad(d \in \mathbb{Z}, i \in \mathbb{Z}) .
$$

The dual of the group $\mathbb{Z}[1 / N]$ is the solenoid

$$
\mathcal{S}_{N}:=\left\{\left(z_{n}\right)_{n \geq 0}\left|z_{n+1}^{N}=z_{n},\right| z_{n} \mid=1 \text { for } n \geq 0\right\} .
$$

Sometimes it will be useful to index the components by $\mathbb{Z}$ and identify $\mathcal{S}_{N}$ with

$$
\mathcal{S}_{N}:=\left\{\left(z_{n}\right)_{n \in \mathbb{Z}}\left|z_{n+1}^{N}=z_{n},\right| z_{n} \mid=1 \text { for } n \in \mathbb{Z}\right\},
$$

the identification being given by

$$
\left(z_{n}\right)_{n \geq 0} \leftrightarrow\left(z_{n}\right)_{n \in \mathbb{Z}} \text {, with } z_{-n}=z_{0}^{N^{n}} \text { for } n>0 .
$$

The duality is given by

$$
\left\langle\frac{k}{N^{p}} \mid\left(z_{n}\right)_{n \in \mathbb{Z}}\right\rangle=z_{p}^{k} \quad\left(\frac{k}{N^{p}} \in \mathbb{Z}[1 / N],\left(z_{n}\right)_{n \in \mathbb{Z}} \in \mathcal{S}_{N}\right) .
$$

The dual action, $\hat{\alpha}$ of $\mathbb{Z}$ on $\mathcal{S}_{N}$, is given by the shift $S$ on $\mathcal{S}_{N}$ :

$$
S\left(z_{n}\right)_{n \in \mathbb{Z}}=\left(z_{n-1}\right)_{n \in \mathbb{Z}}, \quad \hat{\alpha}_{i}\left(z_{n}\right)_{n \in \mathbb{Z}}=S^{i}\left(z_{n}\right)_{n \in \mathbb{Z}} \quad\left(\left(z_{n}\right)_{n \in \mathbb{Z}} \in \mathcal{S}_{N}\right) .
$$

The dual of the inclusion $i: \mathbb{Z}[1 / N] \rightarrow \mathbb{R}$ is $\hat{i}: \mathbb{R} \rightarrow \mathcal{S}_{N}$,

$$
\hat{i}(x)=\left(e^{-2 \pi \mathrm{i} N^{-n} x}\right)_{n \in \mathbb{Z}}, \quad(x \in \mathbb{R}) .
$$

If $\pi$ is a unitary representation of the group $B S(1, N)$ on some Hilbert space we will use capital letters to denote the corresponding operators: $\pi(u)=: U, \pi(t)=: T$, $\pi\left(t_{d}\right)=T_{d}$ for $d \in \mathbb{Z}[1 / N]$.

Representations of the Baumslag Solitar group that admit wavelet bases can also be constructed on some other spaces such as $L^{2}(\mathbb{R}) \oplus \ldots \oplus L^{2}(\mathbb{R})$ (BDP, Dut2] or some fractal spaces ([DutJo $)$.

Here we define the concepts of wavelet theory in the abstract setting, when we have a representation of the group $B S(1, N)$ on some Hilbert space.

Let $\pi$ be a representation of the group $B S(1, N)$ on some Hilbert space $H$. A wavelet for the representation $\pi$ is a finite set $\left\{\psi_{1}, \ldots, \psi_{n}\right\}$ of vectors in $H$ such that

$$
\left\{\pi\left(u^{j} t^{k}\right) \psi_{i} \mid j, k \in \mathbb{Z}, i \in\{1, \ldots, n\}\right\}
$$

is an orthonormal basis for $H$.

We will see in section 2 that the representations that admit wavelets are faithful and weakly equivalent to the right regular representation of the group. 
The main technique used to construct wavelets is a multiresolution analysis, which is a sequence of subspaces $\left(V_{n}\right)_{n \in \mathbb{Z}}$ with the following properties:

(i) $V_{n} \subset V_{n+1} \quad(n \in \mathbb{Z})$;

(ii) $U V_{n+1}=V_{n} \quad(n \in \mathbb{Z})$;

(iii) $\bigcup V_{n}$ is dense in $H$;

(iv) $\cap V_{n}=\{0\}$;

(v) there exists $\varphi$ in $V_{0}$ such that $\left\{T^{k} \varphi \mid k \in \mathbb{Z}\right\}$ is an orthonormal basis for $V_{0}$.

Such a vector $\varphi$ is called an orthogonal scaling vector. For the details of the multiresolution construction we refer to Dau92] and BDP]. Here we recall some facts.

If a multiresolution is given, the wavelets can be constructed by looking for $N-1$ functions $\psi_{1}, \ldots, \psi_{N-1}$ such that

$$
\left\{T^{k} \psi_{i} \mid k \in \mathbb{Z}, i \in\{1, \ldots, N-1\}\right\}
$$

is an orthonormal basis for $V_{1} \ominus V_{0}$.

If the spectral measure of the operator $T=\pi(t)$ is absolutely continuous with respect to the Lebesgue measure on $\mathbb{T}:=\{z \in \mathbb{C}|| z \mid=1\}$, then, by Borel functional calculus, we can define a representation of $L^{\infty}(\mathbb{T})$ on $H$ by

$$
\pi(f)=f(T) \quad\left(f \in L^{\infty}(\mathbb{T})\right),
$$

and this representation will satisfy

$$
U \pi(f) U^{-1}=\pi\left(f\left(z^{N}\right)\right) \quad\left(f \in L^{\infty}(\mathbb{T})\right), \quad \pi(z)=T .
$$

(On $\mathbb{T}$, we have the Lebesgue measure $\mu$.)

Since $U \varphi \in V_{-1} \subset V_{0}$ the scaling vector $\varphi$ satisfies the following scaling equation:

$$
U \varphi=\sum_{k \in \mathbb{Z}} a_{k} T^{k} \varphi
$$

for some coefficients $a_{k} \in \mathbb{C}$. This can be rewritten as

$$
U \varphi=\pi\left(m_{0}\right) \varphi
$$

with $m_{0}(z)=\sum_{k \in \mathbb{Z}} a_{k} z^{k}, z \in \mathbb{T} . m_{0}$ is called the low-pass filter.

A vector $\varphi$ that satisfies (1.3) for some $m_{0} \in L^{\infty}(\mathbb{T})$ and that is cyclic for the representation of the group is called a scaling vector with filter $m_{0}$.

Each pair of vectors $v_{1}, v_{2}$ has a correlation function $h_{v_{1}, v_{2}} \in L^{1}(\mathbb{T})$ associated to it. This is defined by

$$
\left\langle T^{k} v_{1} \mid v_{2}\right\rangle=\int_{\mathbb{T}} z^{k} h_{v_{1}, v_{2}} d \mu \quad(k \in \mathbb{Z}),
$$

or, equivalently, by the Radon-Nikodym derivative of the functional

$$
f \mapsto\left\langle\pi(f) v_{1} \mid v_{2}\right\rangle \text {. }
$$

We denote $h_{v}:=h_{v, v}$.

If $\varphi$ is a scaling vector with filter $m_{0}$, then its correlation function satisfies the following equation:

$$
R_{m_{0}} h_{\varphi}=h_{\varphi}
$$

where $R_{m_{0}}$ is the Ruelle operator defined on $L^{1}(\mathbb{T})$ by

$$
R_{m_{0}} f(z)=\frac{1}{N} \sum_{w^{N}=z}\left|m_{0}(w)\right|^{2} f(w) \quad\left(f \in L^{1}(\mathbb{T}), z \in \mathbb{T}\right) .
$$


The interesting fact is that the converse is also true, in the sense that each scaling equation (1.3) has a solution in some Hilbert space such that the correlation function of the scaling vector is some prescribed function $h$ with $R_{m_{0}} h=h$. More precisely, we have:

Theorem 1.1 ([Jor01, Dut3]). Let $m_{0} \in L^{\infty}(\mathbb{T})$ and $h \in L^{1}(\mathbb{T})$ such that $h \geq 0$ and $R_{m_{0}} h=h$. Then there exist a representation $\pi$ of the group $B S(1, N)$ on a Hilbert space $H$ and $\varphi \in H$ such that

$$
U \varphi=\pi\left(m_{0}\right) \varphi,
$$

and the correlation function of $\varphi$ is h. Moreover, this is unique up to isomorphism.

We call this representation the wavelet representation associated to $m_{0}$ and $h$.

If $h=1$, then the scaling vector is an orthogonal one and $m_{0}$ satisfies the relation

$$
R_{m_{0}} 1=1
$$

which is known in the literature as the quadrature mirror filter (QMF) equation.

Our goal is to analyze these representation, perform the direct integral decomposition and evaluate the consequences that these have on the scaling equation.

In section 3 we construct the theoretical framework for this purpose. We define a special type of representation (Definition 3.1), and we prove in section 4 that the wavelet representations are a particular case (Theorem 4.3). We classify these representation (Proposition 3.3) and give a criterion that identifies them among the representations of the Baumslag Solitar group (Proposition 3.4).

In section 4 we establish a connection between wavelet representation and the measures associated to some random walks on $\mathbb{T}$ which were introduced in a recent paper by Palle Jorgensen Jor04. Theorem 4.3 is the central result of the paper, and it shows that the wavelet representations can be realized on the solenoid $\mathcal{S}_{N}$ using the measure associated to a random walk. Corollary [4.6 establishes the 1-1 correspondence between operators in the commutant, functions which are invariant for the shift $S$, and fixed points of the transfer operator $R_{m_{0}}$.

In Theorem 4.7 we give the direct integral decomposition of the wavelet representation which corresponds to the decomposition of the random walk measure into ergodic components.

Section 5 is dedicated to examples. In section 5.1 we analyze the representation on $L^{2}(\mathbb{R}) \oplus \ldots \oplus L^{2}(\mathbb{R})$ (which obviously contains the classical case on $L^{2}(\mathbb{R})$ ). We describe the direct integral decomposition (Theorem 5.1). This generalizes the result from [LPT]. We also describe the associated measure $m$ on the solenoid extending in this way some results from [Jor04.

As some consequences of the decomposition we mention Theorem 5.4 and Corollary [5.5 which give general necessary and sufficient conditions for the existence of $L^{2}(\mathbb{R})$-solutions of the scaling equation and which can also be used as some ergodic statements about QMF filters.

In section 5.2 we deal with the representation on a fractal measure that was introduced in DutJo. We compute the Fourier coefficients of the associated measure (Proposition 5.6), which gives also a geometric insight into the Cantor set (Lemma 5.7), and we propose a Fourier transform for this fractal measure (Corollary [5.8).

The last example is for $m_{0}=1$ when we reobtain the Haar measure on $\mathcal{S}_{N}$ and its ergodic properties relative to the shift which imply the irreducibility of the representation. 


\section{Representations that haVe WAVELEtS}

In this section we analyze some restrictions that are imposed on a representation of the Baumslag Solitar group $B S(1, N)$ in the case when this representation has a wavelet.

It is known that the existence of a wavelet establishes some strong restrictions on the representation. Some of these restrictions are analyzed in Web for each of the operators $U$ and $T$ in part. For example, it is shown that $U$ and $T$ must both be bilateral shifts of infinite multiplicity. Here we analyze $U$ and $T$ coupled by the commuting relation $U T U^{-1}=T^{N}$.

The next result generalizes Theorem 5.1 in [MV00], but the proof follows the same ideas.

Theorem 2.1. Let $\pi$ be a representation of the group $B S(1, N)$ that has a wavelet. Then $\pi$ extends to a faithful representation of $C_{r}^{*}(B S(1, N))$, the reduced $C^{*}$-algebra of $B S(1, N)$, i.e., the $C^{*}$-algebra generated by the right regular representation. Each element in the $C^{*}$-algebra generated by $\pi$ has a connected spectrum.

Proof. Let $\left\{\psi_{1}, \ldots, \psi_{p}\right\}$ be an orthonormal wavelet. Since the group is amenable, $\pi$ is weakly contained in the right regular representation. For the converse, take $g \in B S(1, N)$. Then $g$ is of the form $g=u^{j} t_{\lambda}$ for some $j \in \mathbb{Z}$ and some $\lambda \in \mathbb{Z}[1 / N]$. Then, for $k \in \mathbb{Z}$ we have

$$
\left\langle\pi(g) \pi\left(u^{-k}\right) \psi_{1} \mid \pi\left(u^{-k}\right) \psi_{1}\right\rangle=\left\langle\pi\left(u^{j} u^{k} t_{\lambda} u^{-k}\right) \psi_{1} \mid \psi_{1}\right\rangle=\left\langle\pi\left(u^{j} t_{\lambda N^{k}}\right) \psi_{1} \mid \psi_{1}\right\rangle .
$$

But, for $k$ big enough, $\lambda N^{k}$ is an integer $l$, and therefore

$$
\left\langle\pi(g) \pi\left(u^{-k}\right) \psi_{1} \mid \pi\left(u^{-k}\right) \psi_{1}\right\rangle=\left\langle\pi\left(u^{j} t^{l}\right) \psi_{1} \mid \psi_{1}\right\rangle=\delta_{e, g}
$$

This implies that the right regular representation is weakly contained in $\pi$, and the first assertion is proved.

Since the representation is faithful and the spectrum is invariant under isomorphisms, the last assertion follows from Lemma 2.1 in MV00.

\section{A CLASS OF IRREDUCIBLE REPRESENTATIONS}

For a measure $\nu$ on $\mathcal{S}_{N}$, we denote by $\nu \circ S$ the measure defined by $\nu \circ S(E)=$ $\nu(S(E))$ for all measurable subsets $E$ of $\mathcal{S}_{N}$. Alternatively, for $f$ measurable and positive on $\mathcal{S}_{N}$,

$$
\int_{\mathcal{S}_{N}} f d \nu \circ S=\int_{\mathcal{S}_{N}} f \circ S^{-1} d \nu .
$$

Definition 3.1. Let $\nu$ be a $\sigma$-finite Borel measure on $\mathcal{S}_{N}$ such that $\nu \circ S$ and $\nu$ are mutually absolutely continuous; we say that $\nu$ is quasi-invariant for $S$. Also, consider a measurable map $\theta: \mathcal{S}_{N} \rightarrow \mathbb{T}$. Let

$$
\Delta:=\frac{d(\nu \circ S)}{d \nu} \text {. }
$$

Define $H=L^{2}(\nu)$,

$$
\begin{gathered}
U \xi=\sqrt{\Delta} \theta \xi \circ S \quad\left(\xi \in L^{2}(\nu)\right) \\
T \xi\left(z_{n}\right)_{n \in \mathbb{Z}}=z_{0} \xi\left(z_{n}\right)_{n \in \mathbb{Z}} \quad\left(\xi \in L^{2}(\nu),\left(z_{n}\right)_{n \in \mathbb{Z}} \in \mathcal{S}_{N}\right) .
\end{gathered}
$$

Proposition 3.2. $\quad$ (i) With $\nu$ and $\theta$ as in Definition 3.1, $U$ and $T$ define a representation $\pi_{\nu, \theta}$ of the group $B S(1, N)$. 
(ii) If $f$ is in $L^{\infty}(\nu)$ and $n \in \mathbb{Z}$, then

$$
U^{n} M_{f} U^{-n}=M_{f \circ S^{n}},
$$

where, for $g \in L^{\infty}(\nu), M_{g}$ is the multiplication operator by $g, M_{g} \xi=g \xi$. For $n, k \in \mathbb{Z}, U^{-n} T^{k} U^{n}$ is the multiplication by the character $k / N^{n}$.

The von Neumann algebra generated by $\left\{U^{n} T^{k} U^{-n} \mid k, n \in \mathbb{Z}\right\}$ is

$$
\left\{M_{f} \mid f \in L^{\infty}(\nu)\right\} \text {. }
$$

(iii) The commutant of the representation is given by

$$
\left\{M_{f} \mid f \in L^{\infty}(\nu), f \circ S=f, \nu \text {-a.e. }\right\},
$$

so the representation is irreducible if and only if $S$ is ergodic with respect to $\nu$, i.e., the sets $A$ which are invariant with respect to $S$ have 0 or full measure.

Proof. Since $\Delta=d(\nu \circ S) / d \nu$, it follows that

$$
\int_{\mathcal{S}_{N}} \xi d \nu=\int_{\mathcal{S}_{N}} \Delta \xi \circ S d \nu \quad\left(\xi \in L^{1}(\nu)\right),
$$

and this implies that $U$ is an isometry. Since $\nu$ is also absolutely continuous with respect to $\nu \circ S$, the inverse of $U$ is well defined by

$$
U^{-1} \xi=\frac{1}{\theta \circ S^{-1} \sqrt{\Delta} \circ S^{-1}} \xi \circ S^{-1} \quad\left(\xi \in L^{2}(\nu)\right),
$$

and therefore $U$ is unitary. $T$ is just a multiplication operator by a function which has absolute value 1 , so $T$ is also unitary.

Now take $n, k \in \mathbb{Z}$ and compute by induction

$$
U^{n} \xi=(\theta \sqrt{\Delta})^{(n)} \xi \circ S^{n},
$$

where, for a function $f$ on $\mathcal{S}_{N}$ we use the notation

$$
f^{(n)}=\left\{\begin{array}{ccc}
f \circ S \ldots f \circ S^{n-1} & \text { if } & n>0, \\
1 & \text { if } & n=0, \\
\frac{1}{f \circ S^{-1} \ldots f \circ S^{-n}} & \text { if } & n<0 .
\end{array}\right.
$$

Then, after a straightforward computation, we obtain

$$
U^{-n} T^{k} U^{n} \xi\left(z_{i}\right)_{i \in \mathbb{Z}}=z_{n}^{k} \xi\left(z_{i}\right)_{i \in \mathbb{Z}},
$$

and if $f$ is in $L^{\infty}\left(\mathcal{S}_{N}\right)$, then

$$
U^{n} M_{f} U^{-n}=M_{f \circ S^{n}} .
$$

In particular $U T U^{-1}=T^{N}$, so this is indeed a representation of $B S(1, N)$.

The proof of the last statement in (ii) is a standard argument of the duality theory: by Pontrjagin's duality theory, the linear span of characters $k / N^{n}$ is uniformly dense in $C\left(\mathcal{S}_{N}\right)$ and any function in $L^{\infty}(\nu)$ can be approximated pointwise $\nu$ a.e. by continuous ones, so the von Neumann algebra generated by the operators of multiplication by characters is $L^{\infty}\left(\mathcal{S}_{N}\right)$ (we will use the identification between a function $f \in L^{\infty}\left(\mathcal{S}_{N}\right)$ and the multiplication operator $\left.M_{f}\right)$.

It remains to compute the commutant. If $S$ is an operator that commutes with $U$ and $T$, it must commute with the entire von Neumann algebra generated by the elements of the form $U^{n} T^{k} U^{-n}$, and we saw that this is $L^{\infty}(\nu)$. Since this algebra is maximal abelian, $S$ must belong to it, so $S=M_{f}$ for some $f \in L^{\infty}(\nu)$. However, $S$ must commute with $U$, too, so $M_{f}=U M_{f} U^{-1}=M_{f \circ S}$. Therefore $f=f \circ S$. 
$S$ is ergodic if and only if the only such functions are the ones that are constant $\nu$-a.e. In conclusion, the commutant is trivial, and the representation is irreducible if and only if $S$ is ergodic.

Proposition 3.3. Let $\left(\nu_{1}, \theta_{1}\right)$ and $\left(\nu_{2}, \theta_{2}\right)$ be as in Definition [3.1, and let $U_{1}, T_{1}$ and $U_{2}, T_{2}$ be the corresponding representations. The representations are equivalent if and only if $\nu_{1}$ and $\nu_{2}$ are mutually absolutely continuous and $\theta_{1}$ and $\theta_{2}$ are cocycle equivalent, in the sense that there exists a function $\lambda: \mathcal{S}_{N} \rightarrow \mathbb{T}$ such that

$$
\lambda \theta_{1}=\lambda \circ S \theta_{2}, \nu_{1}-\text { a.e. }
$$

Proof. Suppose the representations are equivalent and let $W: L^{2}\left(\nu_{1}\right) \rightarrow L^{2}\left(\nu_{2}\right)$ be an intertwining isomorphism. Then, restricting our attention to $\mathbb{Z}[1 / N]$, we see that $W$ establishes the equivalence between the two representations of the abelian algebra $C\left(\mathcal{S}_{N}\right)$ by multiplication operators on $\nu_{1}$ and $\nu_{2}$. This implies that $\nu_{1}$ and $\nu_{2}$ are mutually absolutely continuous.

Let $\eta:=d \nu_{1} / d \nu_{2}$. The operator $\tilde{W}: L^{2}\left(\nu_{1}\right) \rightarrow L^{2}\left(\nu_{2}\right)$ defined by

$$
\tilde{W} \xi_{1}=\sqrt{\eta} \xi_{1} \quad\left(\xi_{1} \in L^{\infty}\left(\nu_{1}\right)\right)
$$

is an isomorphism. Then, $\tilde{W} W^{*}$ is a unitary operator which commutes with $L^{\infty}\left(\nu_{2}\right)$. Therefore, as this is a maximal abelian subalgebra, $\tilde{W} W^{*}=M_{\lambda}$ for some function $\lambda$ on $\mathcal{S}_{N}$ which has absolute value 1 a.e. Then $W=M_{\bar{\lambda}} M_{\sqrt{\eta}}$.

Since $W U_{1}=U_{2} W$, we get for $\xi \in L^{2}\left(\nu_{1}\right)$,

$$
\bar{\lambda} \sqrt{\eta} \theta_{1} \sqrt{\Delta_{1}} \xi_{1} \circ S=\theta_{2} \sqrt{\Delta_{2}} \bar{\lambda} \circ S \sqrt{\eta \circ S} \xi_{1} \circ S .
$$

But

$$
\eta \circ S=\frac{d \nu_{1} \circ S}{d \nu_{2} \circ S}=\frac{d \nu_{1} \circ S}{d \nu_{1}} \frac{d \nu_{1}}{d \nu_{2}} \frac{d \nu_{2}}{d \nu_{2} \circ S}=\Delta_{1} \eta \frac{1}{\Delta_{2}},
$$

so $\bar{\lambda} \theta_{1}=\bar{\lambda} \circ S \theta_{2}, \nu_{1}$-almost everywhere.

For the converse, take $\eta=d \nu_{1} / d \nu_{2}$ and define $W$ from $L^{2}\left(\nu_{1}\right)$ to $L^{2}\left(\nu_{2}\right)$ by

$$
W \xi_{1}=\lambda \sqrt{\eta} \xi_{1} \quad\left(\xi_{1} \in L^{2}\left(\nu_{1}\right)\right)
$$

This defines an isomorphism which clearly intertwines $T_{1}$ and $T_{2}$, and using equation (3.3), we see that it also intertwines $U_{1}$ and $U_{2}$.

Proposition 3.4. Let $\pi$ be a representation of the group $B S(1, N)$ such that the restriction of the representation to the subgroup $\mathbb{Z}[1 / N]$ has a cyclic vector. Then there is a quasi-invariant probability measure $\nu$ on $\mathcal{S}_{N}$, and a map $\theta: \mathcal{S}_{N} \rightarrow \mathbb{T}$ such that $\pi$ is equivalent to the representation $\pi_{\nu, \theta}$ (see Proposition [3.2).

Proof. By the Stone-Mackey theorem applied to the abelian group $\mathbb{Z}[1 / N]$, we can find a measure $\nu$ on the dual group $\mathcal{S}_{N}$ and a measurable multiplicity function $m$ : $\mathcal{S}_{N} \rightarrow\{0,1, \ldots, \infty\}$ such that there is an isometric isomorphism $\Phi$ from the Hilbert space of the representation $H$ to $L^{2}\left(\mathcal{S}_{N}, \nu, m\right):=\bigoplus_{j \geq 1} L^{2}\left(\left\{z \in \mathcal{S}_{N} \mid m(z) \geq j\right\}, \nu\right)$ which transforms the representation into multiplication operators,

$$
\left(\Phi \pi(\lambda) \Phi^{-1}\right)\left(\xi_{j}\right)_{j \geq 1}=\left(\chi_{\lambda} \xi_{j}\right)_{j \geq 1} \quad\left(\left(\xi_{j}\right)_{j} \in L^{2}\left(\mathcal{S}_{N}, \nu, m\right)\right) .
$$

Here $\chi_{\lambda}$ is the character of the group $\mathcal{S}_{N}$ given by the duality in (1.1):

$$
\chi_{\lambda}\left(\left(z_{n}\right)_{n \in \mathbb{Z}}\right)=\left\langle\lambda \mid\left(z_{n}\right)_{n \in \mathbb{Z}}\right\rangle \quad\left(\left(z_{n}\right)_{n \in \mathbb{Z}} \in \mathcal{S}_{N}\right) .
$$

Since there is a cyclic vector, the multiplicity function can be taken to be constant 1 , and we can take $\nu\left(\mathcal{S}_{N}\right)=1$. Thus the representation of $\mathbb{Z}[1 / N]$ is equivalent to 
the representation on $L^{2}(\nu)$ by multiplication by characters, and $\Phi \pi(\lambda) \Phi^{-1}=M_{\chi_{\lambda}}$, for $\lambda \in \mathbb{Z}[1 / N]$. So (by composition with the isomorphism $\Phi$ ) we can assume that the representation $\pi$ is on $L^{2}(\nu)$ and $\pi(\lambda)=M_{\chi_{\lambda}}$.

The representation $\pi(u)$ of the other generator $u$ of the group $B S(1, N)$ is a unitary $U$ with the property

$$
U \pi(\lambda) U^{-1}=\pi\left(u \lambda u^{-1}\right)=\pi(N \lambda) \quad(\lambda \in \mathbb{Z}[1 / N]) .
$$

But this implies, by approximation, that

$$
U M_{f} U^{-1}=M_{f \circ S} \quad\left(f \in L^{\infty}(\nu)\right) .
$$

Then $U \xi=\xi \circ S U 1$, for $\xi \in L^{\infty}(\nu)$. Denote $f:=U 1$. Then, since $U$ is unitary, we have that, for $\xi \in L^{\infty}(\nu)$,

$$
\int_{\mathcal{S}_{N}}\left|\xi \circ S^{-1}\right|^{2} d \nu=\int_{\mathcal{S}_{N}}|f|^{2}|\xi|^{2} d \nu,
$$

so $d(\nu \circ S) / d \nu=|f|^{2}$, and $f$ does not vanish on a set of positive measure, so $\nu$ is quasi-invariant. Take $\theta=f /|f|$, and everything follows.

Example 3.5. Take a point $x=\left(z_{i}\right)_{i \in \mathbb{Z}} \in \mathcal{S}_{N}$ and let $\nu$ be the counting measure on the orbit on this point under $S$ :

$$
\nu(E)=\operatorname{card}\left(\left\{S^{n}(x) \mid n \in \mathbb{Z}\right\} \cap E\right) \quad\left(E \subset \mathcal{S}_{N}\right) .
$$

Take $\theta=1$.

$\nu$ and $\theta$ satisfy the requirements of Proposition 3.2 and let $U, T$ be the corresponding representation. This representation is irreducible because $S$ is ergodic with respect to the measure $\nu$. We distinguish two cases:

(i) If $x$ is periodic (which means that the orbit is finite), i.e., there exists $p>0$ such that $z_{i+p}=z_{i}$ for all $i \in \mathbb{Z}$, then we can take $p$ minimal with this property, and we see that the representation is equivalent to the following on $H_{x}=\mathbb{C}^{p}$ :

$$
\begin{gathered}
U_{x}\left(\xi_{0}, \ldots, \xi_{p-1}\right)=\left(\xi_{p-1}, \xi_{0}, \ldots, \xi_{p-2}\right), \\
T_{x}\left(\xi_{0}, \ldots, \xi_{p-1}\right)=\left(z_{0} \xi_{0}, z_{1} \xi_{1}, \ldots z_{p-1} \xi_{p-1}\right) .
\end{gathered}
$$

(ii) If $x$ is not periodic, then the representation can be realized on $l^{2}(\mathbb{Z})$, with

$$
\begin{gathered}
U_{x} \xi(k)=\xi(k-1) \quad(k \in \mathbb{Z}), \\
T_{x} \xi(k)=z_{k} \xi(k) \quad(k \in \mathbb{Z}) .
\end{gathered}
$$

The representations associated to two points $x, x^{\prime} \in \mathcal{S}_{N}$ are equivalent if and only if $x$ and $x^{\prime}$ are on the same orbit.

These representations can also be obtained using the Mackey machine (see for example chapter 6 of [Fol] ) as some induced representations. However, the technique of Mackey does not give all the irreducible representations because the action of $\mathbb{Z}$ on $\mathbb{Z}[1 / N]$ is not regular (see Theorem 6.42 in [Fol]). One example of an irreducible representation that does not come frm the Mackey construction is given next.

Example 3.6. Consider $\mu_{\mathcal{S}_{N}}$ to be the Haar measure on $\mathcal{S}_{N}$. By the uniqueness of the Haar measure, $\mu_{\mathcal{S}_{N}} \circ S=\mu_{\mathcal{S}_{N}}$. By a theorem of Rohlin-Halmos (see [Wal]), $S$ is ergodic with respect to the Haar measure if and only if the only character $\lambda=k / N^{p}$ satisfying $\alpha_{n}(\lambda)=\lambda$ for some $n \in \mathbb{Z} \backslash\{0\}$ is the trivial one. But this is clear because $\alpha_{n}(\lambda)=N^{n} \lambda$. 
This implies that the representation associated to the Haar measure as in Definition 3.1 is irreducible. We will see that this is actually a wavelet representation associated to the filter $m_{0}=1$.

\section{WAVELET REPRESENTATIONS AND RANDOM WALKS}

In a recent paper Jor04, Palle Jorgensen, extending some earlier work by Richard Gundy Gun00, has realized a connection between wavelet theory and some probability measures associated to certain random walks. Here we recall the definition of these measures and refer the reader to Jor04 for the details. The measures are perfectly adapted to our purpose, and we prove in Theorem 4.3 that the wavelet representation associated to some filter $m_{0}$ can be realized on such a measure.

Consider the $N$ inverse branches of the map $\sigma: x \mapsto N x \bmod 1$, on $[0,1)$, $\tau_{k}:[0,1) \rightarrow\left[\frac{k}{N}, \frac{k+1}{N}\right)$,

$$
\tau_{k}(x)=\frac{x+k}{N} \quad(x \in[0,1], k \in\{0, \ldots, N-1\}) .
$$

Denote $\Omega:=\{0, \ldots, N-1\}^{\mathbb{N}}$ with the product topology.

Consider a function $W \in L^{\infty}[0,1], W \geq 0$, with the property that

$$
\sum_{k=0}^{N-1} W\left(\tau_{k}(x)\right)=1 \quad(x \in[0,1)) .
$$

For example, if $m_{0} \in L^{\infty}(\mathbb{T})$ satisfies $R_{m_{0}} 1=1$, then $W$ defined by $W(x)=$ $\left|m_{0}\left(e^{-2 \pi \mathrm{i} x}\right)\right|^{2} / N$ will satisfy (4.1).

We can identify functions on $[0,1)$ with functions on $\mathbb{T}$ by

$$
W(x) \leftrightarrow W\left(e^{-2 \pi \mathrm{i} x}\right) .
$$

Also, we can identify functions $f$ on $\mathbb{T}$ with functions on the $\mathcal{S}_{N}$ that depend only on the first coordinate:

$$
f\left(\left(z_{n}\right)_{n \in \mathbb{Z}}\right):=f\left(z_{0}\right) \quad\left(\left(z_{n}\right)_{n \in \mathbb{Z}} \in \mathcal{S}_{N}\right) .
$$

It is proved in Jor04, that for each $x \in[0,1)$ there exists a probability measure $P_{x}$ on $\Omega$ such that, if a function $f$ on $\Omega$ depends only on a finite number of coordinates, $\omega_{1}, \ldots, \omega_{n}$, then

$$
\int_{\Omega} f d P_{x}=\sum_{\omega_{1}, \ldots, \omega_{n}} f\left(\omega_{1}, \ldots, \omega_{n}\right) W\left(\tau_{\omega_{1}} x\right) W\left(\tau_{\omega_{2}} \tau_{\omega_{1}} x\right) \ldots W\left(\tau_{\omega_{n}} \ldots \tau_{\omega_{1}} x\right) .
$$

We can identify the space $[0,1) \times \Omega$ with the solenoid $\mathcal{S}_{N}$ :

Proposition 4.1. The map $\Phi:[0,1) \times \Omega \rightarrow \mathcal{S}_{N}$ defined by

$$
\Phi(x, \omega)=\left(e^{-2 \pi i x}, e^{-2 \pi i \tau_{1} x}, e^{-2 \pi i_{\tau_{2} \tau_{1} x}}, \ldots, e^{-2 \pi i \tau_{n} \ldots \tau_{1} x}, \ldots\right) \quad(x \in[0,1), \omega \in \Omega),
$$

is a measurable bijection.

Note that under this identification, the shift takes the form

$$
\Phi^{-1} S \Phi:(x, \omega) \mapsto\left(\sigma(x), i_{x} \omega_{1} \omega_{2} \ldots\right), \text { where } i_{x}=k \text { if } x \in\left[\frac{k}{N}, \frac{k+1}{N}\right),
$$

and its inverse is

$$
\Phi^{-1} S^{-1} \Phi:(x, \omega) \mapsto\left(\tau_{\omega_{1}}(x), \omega_{2} \omega_{3}, \ldots\right)
$$


Define the measure $m$ on $\mathcal{S}_{N}$ by

$$
\int_{\mathcal{S}_{N}} f d m=\int_{[0,1)} \int_{\Omega} f(\Phi(x, \omega)) d P_{x}(\omega) d x \quad\left(f \in C\left(\mathcal{S}_{N}\right)\right) .
$$

Proposition 4.2. $m$ is a probability measure on $\mathcal{S}_{N}$ with the following properties:

(i) If $f \in L^{1}(m)$ depends only on the first $n$ coordinates $z_{0}, \ldots, z_{n-1}$, then

$$
\int_{\mathcal{S}_{N}} f d m=\int_{\mathbb{T}} \sum_{w^{N^{n}}=z} f\left(w^{N^{n-1}}, w^{N^{n-2}}, \ldots, w^{N}, w\right) W^{(n)}(w) d z,
$$

where $W^{(n)}(z)=W(z) W\left(z^{N}\right) \ldots W\left(z^{N^{n-1}}\right)$.

(ii) $m$ is the unique probability measure on $\mathcal{S}_{N}$ which satisfies the conditions

and

$$
\int_{\mathcal{S}_{N}} f d m=\int_{\mathbb{T}} f(z) d z \quad\left(f \in L^{1}(\mathbb{T})\right)
$$

$$
\int_{\mathcal{S}_{N}} f \circ S^{-1} d m=\int_{\mathcal{S}_{N}} N W f d m \quad\left(f \in L^{1}(m)\right)
$$

(i.e., $d(m \circ S) / d m=N W)$.

(iii) For $f \in L^{1}(m)$, and $n \geq 0$ (also for $n<0$ when $W$ does not vanish on a set of positive measure),

$$
\int_{\mathcal{S}_{N}} f \circ S^{-n} d m=\int_{\mathcal{S}_{N}} N^{n} W^{(n)} f d m .
$$

(iv) If $W(z)=\sum_{k \in \mathbb{Z}} a_{k} z^{k}$, then

$$
\hat{m}(\lambda):=\int_{\mathcal{S}_{N}} \chi_{\lambda} d m=\int_{\mathbb{T}} z^{l} N^{p} W^{(p)} d z \quad\left(\lambda=l / N^{p} \in \mathbb{Z}[1 / N]\right)
$$

(where $\chi_{\lambda}$ is the character on $\mathcal{S}_{N}$ attached to $\lambda$; see (3.4)). Moreover $\hat{m}(k)=\delta_{k}$ for $k \in \mathbb{Z}$ and $\hat{m}$ satisfies the following scaling equation:

$$
\hat{m}(\lambda)=N \sum_{k \in \mathbb{Z}} a_{k} \hat{m}(N \lambda+k) \quad(\lambda \in \mathbb{Z}[1 / N]) .
$$

Proof. If $f \in L^{1}(m)$ depends only on the first $n$ coordinates, then $f \circ \Phi$ depends only on $x$ and $\omega_{1}, \ldots, \omega_{n-1}$. Also,

$$
f \circ \Phi\left(x, \omega_{1}, \ldots, \omega_{n-1}\right)=f\left(w^{N^{n-1}}, \ldots, w\right), \text { with } w=e^{-2 \pi \mathrm{i} \tau_{\omega_{n-1}} \ldots \tau_{\omega_{1}}(x)} .
$$

So (i) follows from (4.2).

Equation (4.4) is clear and, to prove (4.5), take $f \in C\left(\mathcal{S}_{N}\right)$ which depends only on the first $n$ coordinates. Then $f \circ S^{-1}$ depends only on the first $n+1$ coordinates and

$$
\begin{aligned}
\int_{\mathcal{S}_{N}} f \circ S^{-1} d m & =\int_{0}^{1} \sum_{\omega_{1}, \ldots, \omega_{n}} f \circ S^{-1} \circ \Phi\left(x, \omega_{1}, \ldots, \omega_{n}\right) W\left(\tau_{\omega_{1}} x\right) \ldots W\left(\tau_{\omega_{n}} \ldots\left(\tau_{\omega_{1}} x\right)\right) d x \\
& =\int_{0}^{1} \sum_{\omega_{1}, \ldots, \omega_{n}} f \circ \Phi\left(\tau_{\omega_{1}} x, \omega_{2}, \ldots, \omega_{n}\right) W\left(\tau_{\omega_{1}} x\right) \ldots W\left(\tau_{\omega_{n}} \ldots\left(\tau_{\omega_{1}} x\right)\right) d x .
\end{aligned}
$$

If we denote

$$
g(x):=\sum_{\omega_{2}, \ldots, \omega_{n}} f \circ \Phi\left(x, \omega_{2}, \ldots, \omega_{n}\right) W\left(\tau_{\omega_{2}} x\right) \ldots W\left(\tau_{\omega_{n}} \ldots\left(\tau_{\omega_{2}} x\right)\right)
$$


and use the fact that (with a change of variable)

$$
\int_{0}^{1} \sum_{\omega_{1}} W\left(\tau_{\omega_{1}} x\right) g\left(\tau_{\omega_{1}} x\right) d x=\int_{0}^{1} N W(x) g(x) d x,
$$

then we obtain

$$
\begin{array}{rl}
\int_{\mathcal{S}_{N}} & f \circ S^{-1} d m \\
\quad= & \int_{0}^{1} N W(x) \sum_{\omega_{2}, \ldots, \omega_{n}} f \circ \Phi\left(x, \omega_{2}, \ldots, \omega_{n}\right) W\left(\tau_{\omega_{2}} x\right) \ldots W\left(\tau_{\omega_{n}} \ldots\left(\tau_{\omega_{2}} x\right)\right) d x \\
\quad= & \int_{\mathcal{S}_{N}} N W f d m .
\end{array}
$$

(iii) follows from (ii) by induction. All functions on $\mathcal{S}_{N}$ can be approximated by functions of the form $f \circ S^{-n}$ with $f \in L^{1}(\mathbb{T})$ and $n \geq 0$ (these are the functions which depend only on the first $n$ coordinates). If a measure $m^{\prime}$ satisfies the conditions of (ii), then

$$
\begin{array}{rl}
\int_{\mathcal{S}_{N}} & f \circ S^{-1} d m \\
\quad= & \int_{0}^{1} N W(x) \sum_{\omega_{2}, \ldots, \omega_{n}} f \circ \Phi\left(x, \omega_{2}, \ldots, \omega_{n}\right) W\left(\tau_{\omega_{2}} x\right) \ldots W\left(\tau_{\omega_{n}} \ldots\left(\tau_{\omega_{2}} x\right)\right) d x \\
= & \int_{\mathcal{S}_{N}} N W f d m .
\end{array}
$$

Thus the conditions of (ii) determine $m$ uniquely.

For (iv), observe that $\chi_{\lambda} \circ S^{p}=\chi_{N^{p} \lambda}$ for any $\lambda \in \mathbb{Z}[1 / N]$ and any $p \in \mathbb{Z}$. Then, using (iii),

$$
\hat{m}\left(l / N^{p}\right)=\int_{\mathcal{S}_{N}} N^{p} W^{(p)} \chi_{l} d m=\int_{\mathbb{T}} z^{l} N^{p} W^{p} .
$$

From (4.5), with $f=\chi_{\lambda}$, we obtain

$$
\hat{m}(\lambda / N)=\int_{\mathbb{T}} N \sum_{k \in \mathbb{Z}} a_{k} z_{0}^{k} \chi_{\lambda} d m\left(z_{n}\right)_{n \geq 0}=N \sum_{k \in \mathbb{Z}} a_{k} \int_{\mathbb{T}} \chi_{\lambda+k} d m,
$$

which implies (4.7).

Theorem 4.3. Let $m_{0} \in L^{\infty}(\mathbb{T})$ be non-singular (i.e., it does not vanish on a set of positive measure) with $R_{m_{0}} 1=1$. Let $W:=\left|m_{0}\right|^{2} / N$ and $\theta=m_{0} /\left|m_{0}\right|$. Let $m$ be the measure associated to $W$. Then the wavelet representation associated to $m_{0}$ and $h=1$ is the representation of the Baumslag Solitar group $B S(1, N)$ associated to $m$ and $\theta$, i.e., $H=L^{2}(m)$,

$$
\begin{gathered}
U \xi=m_{0} \xi \circ S, \quad \pi(f) \xi=f \xi \quad\left(\xi \in L^{2}(m), f \in L^{\infty}(\mathbb{T})\right), \\
\varphi=1 .
\end{gathered}
$$

The commutant of the representation is

$$
\left\{M_{f} \mid f \in L^{\infty}(m), f \circ S=f \nu \text {-a.e. }\right\} .
$$


Proof. By Proposition 4.2(ii), we know that $d(m \circ S) / d m=N W=\left|m_{0}\right|^{2}$, and since $m_{0}$ is non-singular, we also have that $m$ is absolutely continuous with respect to $m \circ S$. Therefore we can use Proposition [3.2, and we obtain the representation of $B S(1, N)$. With (4.4) we see that, by Borel functional calculus, $T$ generates a representation $\pi$ of $L^{\infty}(\mathbb{T}), \pi(f)=f(T)=M_{f}$ for $f \in L^{\infty}(\mathbb{T})$.

Note that

$$
\begin{gathered}
\langle\pi(f) \varphi \mid \varphi\rangle=\int_{\mathcal{S}_{N}} f d m=\int_{\mathbb{T}} f(z) d z, \\
U \varphi=\pi\left(m_{0}\right) \varphi .
\end{gathered}
$$

Also

$$
U^{-n} \pi(f) U^{n} \varphi=M_{f \circ S^{-n}} \varphi=f \circ S^{-n},
$$

and, because the functions that depend on only finitely many coordinates are dense in $L^{2}(m)$, it follows that $\varphi$ is cyclic for the representation, so it is a scaling vector.

The commutant is obtained from Proposition 3.3 .

Proposition 4.4. The map $E$ from $L^{1}(m)$ to $L^{1}(\mathbb{T})$ defined by

$$
E(f)\left(e^{-2 \pi i x}\right)=\int_{\Omega} f \circ \Phi(x, \omega) d P_{x}(\omega) \quad\left(f \in L^{1}(m), x \in[0,1)\right)
$$

is a well-defined conditional expectation (i.e., $E^{2}=E, E(f) \geq 0$ if $f \geq 0, E(g f)=$ $g E(f)$ if $g \in L^{\infty}(\mathbb{T})$ and $\left.f \in L^{1}(m)\right)$. Moreover,

$$
\int_{\mathcal{S}_{N}} f d m=\int_{\mathbb{T}} E(f) d z \quad\left(f \in L^{1}(m)\right) .
$$

E maps $L^{\infty}(m)$ into $L^{\infty}(\mathbb{T})$ and $\|E(f)\|_{\infty} \leq\|f\|_{\infty}$.

$E$ maps $L^{2}(m)$ into $L^{2}(\mathbb{T})$, and the restriction of $E$ to $L^{2}(m)$ coincides with the projection onto the subspace of functions that depend only $z$ (which can be identified with $\left.L^{2}(\mathbb{T})\right)$.

Proof. Everything can be checked by some straightforward computations.

Proposition 4.5. If $\xi_{1}, \xi_{2} \in L^{2}(m)$, then their correlation function is $h_{\xi_{1}, \xi_{2}}=$ $E\left(\xi_{1} \bar{\xi}_{2}\right)$.

Proof. For $f \in L^{\infty}(\mathbb{T})$ we have

$$
\left\langle\pi f\left(\xi_{1}\right) \mid \xi_{2}\right\rangle=\int_{\mathcal{S}_{N}} f \xi_{1} \bar{\xi}_{2} d m=\int_{\mathbb{T}} E\left(f \xi_{1} \bar{\xi}_{2}\right) d z=\int_{\mathbb{T}} f E\left(\xi_{1} \bar{\xi}_{2}\right) d z .
$$

Corollary 4.6. There is a one-to-one linear and monotone correspondence between the following data:

(i) Operators $S$ in the commutant of $\{U, T\}$.

(ii) Cocycles, i.e., functions $f \in L^{\infty}(m)$ such that $f \circ S=f$.

(iii) Functions $h \in L^{\infty}(\mathbb{T})$ which are harmonic with respect to the Ruelle operator, i.e., $R_{m_{0}} h=h$.

From (i) to (ii) the correspondence is given in Theorem 4.3. From (ii) to (iii), the correspondence is $f \mapsto h=E(f)$. From (iii) to (i) the correspondence is given by Theorem 3.18 in Dut3. 
Proof. Everything follows from Theorem 4.3, Proposition 4.5, Theorem 3.18 in Dut3, and see also Theorem 2.7.1 in [Jor04].

Theorem 4.7. Let $m_{0} \in L^{\infty}(\mathbb{T})$ be a non-singular filter with $R_{m_{0}} 1=1$, and let $(H, U, \pi, \varphi)$ be the wavelet representation associated to $m_{0}$. Then there is a standard measure space $(A, \mathcal{M}, \mu)$, a measurable field $\left\{\mathcal{H}_{a}\right\}$ of Hilbert spaces on $A$, a measurable field $\left\{\pi_{a}\right\}$ of irreducible representations $B S(1, N)$ and a unitary map $\Psi: H \rightarrow \int^{\oplus} \mathcal{H}_{a} d \mu(a)$, such that

(i) $\Psi \pi(x) \Psi^{-1}=\int^{\oplus} \pi_{a}(x)$, for $x \in B S(1, N)$;

(ii) $\Psi \pi^{\prime} \Psi^{-1}$ is the algebra of diagonal operators on $\int^{\oplus} \mathcal{H}_{a} d \mu(a), \pi^{\prime}$ being the commutant of the representation $\pi$.

For almost every $a \in A$ there exists a unique ergodic, quasi-invariant probability measure $\nu_{a}$ on $\mathcal{S}_{N}$ such that $d\left(\nu_{a} \circ S\right) / d \nu_{a}=\left|m_{0}\right|^{2}$, and if $\theta=m_{0} /\left|m_{0}\right|$, then $\pi_{a}$ is equivalent to the representation $\pi_{\nu_{a}, \theta}$. Moreover, for $f \in C\left(\mathcal{S}_{N}\right)$, if $m$ is the measure associated to $m_{0}$ as in Theorem 4.3, then

$$
\int_{\mathcal{S}_{N}} f d m=\int_{A} \int_{\mathcal{S}_{N}} f d \nu_{a} d \mu(a) .
$$

Proof. Since the commutant of the representation, $\pi^{\prime}$, is abelian (Theorem 4.3) the first statements follow from the well-known direct integral decomposition theory for locally compact groups (see Theorem 7.37 and 7.38 in [Fol]).

Now we want to find $\nu_{a}$. We prove first that for almost every $x$ the vector $\Psi \varphi(a)$ is cyclic for the restriction of the representation $\pi_{a}$ to the subgroup $\mathbb{Z}[1 / N]$. Suppose not. Then we can find a subset $E$ of positive measure such that, for $a$ in $E$,

$$
K_{a}:=\overline{\operatorname{span}}\left\{\pi_{a}(\lambda) \Psi \varphi(a) \mid \lambda \in \mathbb{Z}[1 / N]\right\} \neq \mathcal{H}_{a} .
$$

But then we can define a measurable section $\xi: A \rightarrow \int{ }^{\oplus} \mathcal{H}_{a} d \mu(a)$ such that $\xi(a)=0$ for $a \in A \backslash E$, and, for $a \in E, \xi(a)$ has norm 1 and is orthogonal to $K_{a}$. We have that $\xi$ is orthogonal to

$$
\overline{\operatorname{span}}\{\pi(\lambda) \Psi \varphi \mid \lambda \in \mathbb{Z}[1 / N]\},
$$

which contradicts the fact that $\varphi$ is cyclic for $\pi(\mathbb{Z}[1 / N]$ ) (see Theorem 4.3 ).

Since $\Psi \varphi(a)$ is cyclic for the representation $\pi_{a}(\mathbb{Z}[1 / N])$, by Proposition 3.4 there exists a quasi-invariant probability measure $\nu_{a}^{\prime}$ and a map $\theta_{a}$ such that $\pi_{a}$ is equivalent to $\pi_{\nu_{a}^{\prime}, \theta_{a}}$.

Since $U \varphi=\sum_{k \in \mathbb{Z}} a_{k} T^{k} \varphi$ it follows that

$$
\pi_{a}(U) \Psi \varphi(a)=\sum_{k \in \mathbb{Z}} a_{k} \pi_{a}\left(T^{k}\right) \Psi \varphi(a) \text { for almost every } a \in A .
$$

Let $f_{a}:=d\left(\nu_{a}^{\prime} \circ S\right) / d \nu_{a}^{\prime}$. Then we obtain, identifying $\mathcal{H}_{a}$ with $L^{2}\left(\nu_{a}^{\prime}\right)$, that

$$
\theta_{a} \sqrt{f_{a}} \Psi \varphi(a) \circ S=\sum_{k \in \mathbb{Z}} a_{k} z^{k} \Psi \varphi(a)=m_{0} \Psi \varphi(a), \nu_{a} \text {-a.e. }
$$

Since $\Psi \varphi(a)$ is cyclic for $\pi_{a}(\mathbb{Z}[1 / N])$, which are multiplication operators, $\Psi \varphi(a)$ cannot be zero on a set of positive $\nu_{a}^{\prime}$-measure.

Now consider the measure $d \nu_{a}=\frac{1}{C_{a}}|\Psi \varphi(a)|^{2} d \nu_{a}^{\prime}$, where $C_{a}=\int_{\mathcal{S}_{N}}|\Psi \varphi(a)|^{2} d \nu_{a}$. The measures are mutually absolutely continuous. Also

$$
\frac{d\left(\nu_{a} \circ S\right)}{d \nu_{a}}=\frac{d\left(\nu_{a} \circ S\right)}{d\left(\nu_{a}^{\prime} \circ S\right)} \frac{d\left(\nu_{a}^{\prime} \circ S\right)}{d \nu_{a}^{\prime}} \frac{d \nu_{a}^{\prime}}{d \nu_{a}}=|\Psi \varphi(a) \circ S|^{2} f_{a} \frac{1}{|\Psi \varphi(a)|^{2}}=\left|m_{0}\right|^{2} .
$$


A simple computation shows that $\nu_{a}\left(\mathcal{S}_{N}\right)=1$. Since the representation $\pi_{a}$ is irreducible, by Proposition $3.2, S$ is ergodic w.r.t. $\nu_{a}^{\prime}$, and since this is equivalent to $\nu_{a}, S$ is ergodic w.r.t. $\nu_{a}$.

Using (4.8), we obtain

$$
\theta_{a} \frac{\Psi \varphi(a) \circ S}{|\Psi \varphi(a) \circ S|}=\frac{m_{0}}{\left|m_{0}\right|} \frac{\Psi \varphi(a)}{|\Psi \varphi(a)|},
$$

hence $\theta_{a}$ and $\theta$ are cocycle equivalent, and therefore, by Proposition 3.3. the representations $\pi_{\nu_{a}^{\prime}, \theta_{a}}$ and $\pi_{\nu_{a}, \theta}$ are equivalent.

To prove the uniqueness of the measure $\nu_{a}$, take $\rho_{a}$ to be a measure with the same properties. Since they generate equivalent representations, by Proposition 3.3 they must be mutually equivalent. Let $\eta=d \nu_{a} / d \rho_{a}$. Then

$$
\left|m_{0}\right|^{2}=\frac{d\left(\nu_{a} \circ S\right)}{d \nu_{a}}=\frac{d\left(\nu_{a} \circ S\right)}{d\left(\rho_{a} \circ S\right)} \frac{d\left(\rho_{a} \circ S\right)}{d \rho_{a}} \frac{d \rho_{a}}{d \nu_{a}}=\eta \circ S\left|m_{0}\right|^{2} \frac{1}{\eta} .
$$

Therefore $\eta=\eta \circ S, \nu_{a}$ a.e. But $\nu_{a}$ is ergodic so $\eta$ is constant, and, since both measures are probability measures, $\eta=1$ a.e.

For the last equality, it is enough to take $f$ to be $\chi_{\lambda}$ for some $\lambda \in \mathbb{Z}[1 / N]$, because these are uniformly dense in $C\left(\mathcal{S}_{N}\right)$. Then

$$
\begin{gathered}
\int_{\mathcal{S}_{N}} \chi_{\lambda} d m=\langle\pi(\lambda) \varphi \mid \varphi\rangle=\int_{A}\left\langle\pi_{a}(\lambda) \Psi \varphi(a) \mid \Psi \varphi(a)\right\rangle d \mu(a) \\
=\int_{A} \int_{\mathcal{S}_{N}} \chi_{\lambda}|\Psi \varphi(a)|^{2} d \nu_{a}^{\prime} d \mu(a)=\int_{A} \int_{\mathcal{S}_{N}} \chi_{\lambda} d \nu_{a} d \mu(a) .
\end{gathered}
$$

Remark 4.8. Corollary 4.6 shows that the projections in the commutant of the representation correspond to sets which are invariant for the shift. Proposition 3.2 shows that irreducible representations correspond to ergodic measures. Therefore, the direct integral decomposition of the representation into irreducible components corresponds to the direct integral decomposition of the measure $m$ into its ergodic components (see e.g. [GS]). We can use Theorem 1.1 in [GS to obtain more information about the measures $\nu_{a}$ :

(i) For every Borel subset $B$ of $\mathcal{S}_{N}$,

$$
m(B)=\int_{A} \nu_{a}(B) d \mu(a)
$$

(ii) If $a, a^{\prime} \in A$ and $a \neq a^{\prime}$, then the measures $\nu_{a}$ and $\nu_{a}^{\prime}$ are mutually singular.

Proposition 4.9. With the notation of Theorem 4.7, a vector $\varphi^{\prime} \in H$ is cyclic for the representation iff $\Psi \varphi^{\prime}(a) \neq 0$ for almost all $a \in A$.

Proof. A vector $\varphi^{\prime}$ is cyclic for the representation iff it is separating for the commutant. Since the commutant consists of diagonal operators, $\Psi \varphi^{\prime}$ is cyclic iff, $\lambda_{a} \Psi \varphi(a)=0$ for almost all $a$ implies $\lambda_{a}=0$ for almost all $a$. But this is true iff $\Psi \varphi^{\prime}(a) \neq 0$ for almost all $a$.

\section{EXAMPLES}

In this section we analyze some examples in more detail. We are interested in the measure $m$ associated to $m_{0}$ and in the decomposition of the wavelet representation. We also present some interesting consequences. 
5.1. Representations on $L^{2}(\mathbb{R})^{p}$. Let $C$ be a cycle, $C=\left\{z_{0}, \ldots, z_{p-1}\right\}$, i.e., $z_{1}^{N}=$ $z_{2}, z_{2}^{N}=z_{3}, \ldots, z_{p-2}^{N}=z_{p-1}, z_{p-1}^{N}=z_{0}$, a periodic orbit for the map $z \mapsto z^{N}, p$ being the length of the orbit. Let $\left|\alpha_{0}\right|=\ldots=\left|\alpha_{p-1}\right|=1$. In [BDP] and [Dut2], we constructed a representation of $B S(1, N)$ on $L^{2}(\mathbb{R})^{p}$ by

$$
\begin{gathered}
U f(\xi, i)=\alpha_{i} \frac{1}{\sqrt{N}} f\left(\frac{\xi}{N},(i+1) \bmod p\right) \quad\left(f \in L^{2}(\mathbb{R})^{p}, \xi \in \mathbb{R}, i \in\{0, \ldots, p-1\}\right), \\
T f(\xi, i)=z_{i} f(x-1, i) \quad\left(f \in L^{2}(\mathbb{R})^{p}, \xi \in \mathbb{R}, i \in\{0, \ldots, p-1\}\right) .
\end{gathered}
$$

We denote this representation by $\mathfrak{R}_{C, \alpha}$. Since the representations $\mathfrak{R}_{C, \alpha}$ are isomorphic for $C$ fixed and $\alpha$ variable (see [BDP]), we will work mostly with the case when all $\alpha_{i}=1$, and use the notation $\mathfrak{R}_{C}$.

Taking the Fourier transform the representation becomes

$$
\begin{gathered}
\hat{U} f(\xi, i)=\sqrt{N} f(N x,(i+1) \bmod p) \quad\left(f \in L^{2}(\mathbb{R})^{p}, \xi \in \mathbb{R}, i \in\{0, \ldots, p-1\}\right), \\
\hat{T} f(\xi, i)=z_{i} e^{-2 \pi \mathrm{i} x} f(x, i) \quad\left(f \in L^{2}(\mathbb{R})^{p}, \xi \in \mathbb{R}, i \in\{0, \ldots, p-1\}\right) .
\end{gathered}
$$

We will describe the direct integral decomposition of this representation.

Each $x \in \mathbb{R}$ can be identified with an element in $\mathcal{S}_{N}$ by $\hat{i}(x)=\left(e^{-2 \pi \mathrm{i} N^{-k} x}\right)_{k \in \mathbb{Z}}$. Also we can identify the cycle $C$ with an element $z_{C} \in \mathcal{S}_{N}$,

$$
z_{C}:=\left(z_{(-k) \bmod p}\right)_{k \in \mathbb{Z}} \text {. }
$$

Then the product $x_{C}:=z_{C} \hat{i}(x)$ is an element of the group $\mathcal{S}_{N}$, and, as in Example 3.5. we can construct an irreducible representation $\pi_{x_{C}}$ on $l^{2}(\mathbb{Z})$ by

$$
\begin{gathered}
\pi_{x_{C}}(u) \xi(k)=\xi(k-1) \quad\left(\xi \in l^{2}(\mathbb{Z}), k \in \mathbb{Z}\right), \\
\pi_{x_{C}}(t) \xi(k)=z_{(-k) \bmod p} e^{-2 \pi \mathrm{i} N^{-k} x} \xi(k) \quad\left(\xi \in l^{2}(\mathbb{Z}), k \in \mathbb{Z}\right) .
\end{gathered}
$$

Let $E=\left(-N^{p}, 1\right] \cup\left[1, N^{p}\right)$.

Theorem 5.1. Let $C=\left\{z_{0}, \ldots, z_{p-1}\right\}$ be a cycle. The representation $\mathfrak{R}_{C}$ is isometrically isomorphic to the representation on $L^{2}\left(E, l^{2}(\mathbb{Z})\right)$ given by the direct integral $\int_{E}^{\oplus} \pi_{z_{C} x}$

Proof. Define $\Psi$ from $L^{2}(\mathbb{R})^{p}$ to $L^{2}\left(E, l^{2}(\mathbb{Z})\right)$ by

$$
\Psi(f)(x, m)=\sqrt{N^{-m}} f\left(N^{-m} x,(-m) \bmod p\right) \quad\left(f \in L^{2}(\mathbb{R})^{p}, x \in E, m \in \mathbb{Z}\right) .
$$

We claim that $\Psi$ is an isomorphism with inverse

$$
\Psi^{-1}(f)(\xi, i)=\frac{1}{\sqrt{N^{-m}}} f(x, m) \quad\left(f \in L^{2}\left(E, l^{2}(\mathbb{Z})\right), \xi \in \mathbb{R}, i \in\{0, \ldots, p-1\}\right),
$$

where $x \in E$ and $m \in\{0, \ldots, p-1\}$ are uniquely determined by the equations $\xi=N^{-m} x$ and $(-m) \bmod p=i$.

First, we check that $\Psi$ is an isometry

$$
\begin{aligned}
\sum_{i=0}^{p-1} \int_{\mathbb{R}}|f(\xi, i)|^{2} d \xi & =\sum_{i=0}^{p-1} \sum_{l \in \mathbb{Z}} \int_{N^{p l+i} E}|f(\xi, i)|^{2} d \xi \\
& =\sum_{i=0}^{p-1} \sum_{l \in \mathbb{Z}} \int_{E}\left|f\left(N^{p l+i} x, i\right)\right|^{2} N^{p l+i} d x \\
& =\sum_{m \in \mathbb{Z}} \int_{E}\left|f\left(N^{-m} x,(-m) \bmod p\right)\right|^{2} N^{-m} d x
\end{aligned}
$$


The fact that $\Psi^{-1}$ has the given form follows from a one-line computation.

Next we want to see that $\Psi$ intertwines the representations:

$$
\begin{gathered}
\Psi \hat{U} \Psi^{-1}(f)(x, m)=\sqrt{N^{-m}}\left(\hat{U} \Psi^{-1}(f)\right)\left(N^{-m} x,(-m) \bmod p\right) \\
=\sqrt{N^{-m+1}} \Psi^{-1}(f)\left(N^{-m+1} x,(-m+1) \bmod p\right) \\
=f(x, m-1), \\
\Psi \hat{T} \Psi^{-1}(f)(x, m)=\sqrt{N^{-m}}\left(\hat{T} \Psi^{-1}(f)\right)\left(N^{-m} x,(-m) \bmod p\right) \\
=z_{(-m) \bmod p} e^{-2 \pi \mathrm{i} N^{-m} x} \sqrt{N^{-m}} \Psi^{-1}(f)\left(N^{-m} x,(-m) \bmod p\right) \\
=z_{(-m) \bmod p} e^{-2 \pi \mathrm{i} N^{-m} x} f(x, m) .
\end{gathered}
$$

Next, we will try to describe the measure $m$ associated to a filter $m_{0}$ that gives scaling vectors for $\mathfrak{R}_{C}$.

Recall the following theorem from Dut2:

Theorem 5.2. Suppose $m_{0}=\sum_{k \in \mathbb{Z}} a_{k} z^{k}$ is a Lipschitz function with finitely many zeros, with $R_{m_{0}} 1=1$, and let $C_{i}=\left\{z_{0}^{i}, \ldots, z_{p_{i}-1}^{i}\right\}, i \in\{1, \ldots, n\}$, be the $m_{0}$-cycles (assume there is at least one), i.e., $\left|m_{0}\left(z_{j}^{i}\right)\right|=\sqrt{N}$ for all $i, j$. Denote by $\theta_{k}^{i}$ the argument of $z_{k \bmod p_{i}}^{i}\left(e^{-2 \pi i \theta_{k}^{i}}=z_{k}^{i}\right)$, and let $\alpha_{k}^{i}=m_{0}\left(z_{k \bmod p_{i}}\right) / \sqrt{N} \in \mathbb{T}$. Then

$$
\hat{\varphi}_{k}^{i}(x)=\prod_{l=1}^{\infty} \frac{\bar{\alpha}_{k-l}^{i} m_{0}\left(\frac{x}{N^{l}}+\theta_{k-l}^{i}\right)}{\sqrt{N}} \quad\left(x \in \mathbb{R}, k \in\left\{0, \ldots, p_{i}-1\right\}, i \in\{1, \ldots, n\}\right)
$$

defines an orthonormal scaling vector $\varphi=\left(\varphi_{0}^{i}, \ldots, \varphi_{p_{i}-1}\right)_{i=1, n}$ in $\bigoplus_{i=1}^{n} L^{2}(\mathbb{R})^{p_{i}}$ for the representation $\bigoplus_{i=1}^{n} \mathfrak{R}_{C_{i}, \alpha_{i}}$ with

$$
\begin{gathered}
U \varphi=\pi\left(m_{0}\right) \varphi, \\
\left\langle\pi(t)^{k} \varphi \mid \varphi\right\rangle=\delta_{k} \quad(k \in \mathbb{Z}) .
\end{gathered}
$$

The last equality can be rewritten as

$$
\sum_{i=1}^{n} \sum_{a \in \mathbb{Z}} \sum_{k=1}^{p_{i}}\left|\hat{\varphi}_{k}^{i}\left(x+a-\theta_{k}^{i}\right)\right|^{2}=1 \quad(x \in[0,1]) .
$$

Note that for each $x \in[0,1], a \in \mathbb{Z}$ and $k \in\left\{0, \ldots, p_{i}-1\right\}$, a straightforward calculation based on the fact that $N \theta_{l}^{i} \equiv \theta_{l+1}^{i} \bmod 1$ shows that

$$
z(x, i, a, k):=\left(e^{-2 \pi i\left(\frac{x+a-\theta_{k}^{i}}{N^{l}}+\theta_{k-l}^{i}\right)}\right)_{l \in \mathbb{Z}}=S^{-k}\left(z_{C_{i}}\right) \hat{i}\left(x+a-\theta_{k}^{i}\right) \in \mathcal{S}_{N},
$$

and $\Phi^{-1}(z(x, i, a, k))$ is of the form $(x, \omega(x, i, a, k))$.

Proposition 5.3. If $m_{0}$ satisfies the hypotheses of Theorem [5.2, with the previous assumptions and notations, for all $x \in[0,1]$, the measure $P_{x}$ is atomic, concentrated on

$$
\begin{gathered}
\left\{\omega(x, i, a, k) \mid a \in \mathbb{Z}, k \in\left\{0, \ldots, p_{i}-1\right\}, i \in\{1, \ldots, n\}\right\}, \\
P_{x}(\omega(x, i, a, k))=\left|\hat{\varphi}_{k}^{i}\left(x+a-\theta_{k}^{i}\right)\right|^{2} \quad\left(a \in \mathbb{Z}, k \in\left\{0, \ldots, p_{i}-1\right\}, i \in\{1, \ldots, n\}\right) .
\end{gathered}
$$

The measure $m$ is supported on $\bigcup_{C} \bigcup_{k=0}^{p_{i}-1} S^{-k} z_{C} \hat{i}(\mathbb{R})$. 
Proof. To evaluate the measure of the set $\{\omega(x, i, a, k))\}$, we write it as an intersection of cylinders in $\Omega$ and, using (4.2) and taking the limit, we obtain that

$$
\begin{aligned}
P_{x}(\omega(x, i, a, k))=\prod_{l=1}^{\infty} W\left(z(x, i, a, k)_{l}\right)=\prod_{l=1}^{\infty} \frac{\left|m_{0}\left(\frac{x+a-\theta_{k}^{i}}{N^{l}}+\theta_{k-l}^{i}\right)\right|^{2}}{N} \\
=\left|\hat{\varphi}_{k}^{i}\left(x+a-\theta_{k}^{i}\right)\right|^{2} .
\end{aligned}
$$

But, using (5.2), we obtain that

$$
\sum_{i=1}^{n} \sum_{a \in \mathbb{Z}} \sum_{k=1}^{p_{i}} P_{x}(\omega(x, i, a, k))=1,
$$

and the rest follows.

We can use the decomposition Theorem 5.1 to obtain an interesting ergodic result for quadrature mirror filters.

Theorem 5.4. Let $C=\left\{z_{0}, \ldots, z_{p-1}\right\}$ be a cycle and denote by $\theta_{k}$ the argument of $z_{k} \bmod p$, i.e., $e^{-2 \pi i \theta_{k}}=z_{k \bmod p}$. Let $m_{0} \in L^{\infty}(\mathbb{T})$ such that there exists some (not necessarily orthogonal) scaling vector $\varphi$ cyclic for the representation $\mathfrak{R}_{C}$ with

$$
U \varphi=\pi\left(m_{0}\right) \varphi .
$$

Then for almost every $x \in\left(-N^{p}, 1\right] \cup\left[1, N^{p}\right)$, there exists $k_{x} \in \mathbb{Z}$ such that

$$
\begin{gathered}
\sum_{n=-\infty}^{k_{x}-1} \prod_{k=n-1}^{k_{x}}\left|m_{0}\left(\frac{x}{N^{k}}+\theta_{-k}\right)\right|^{2}<\infty, \\
\sum_{n=k_{x}+1}^{\infty} \frac{1}{\prod_{k=k_{x}+1}^{n}\left|m_{0}\left(\frac{x}{N^{k}}+\theta_{-k}\right)\right|^{2}}<\infty .
\end{gathered}
$$

Conversely, if $m_{0} \in L^{\infty}(\mathbb{T})$ satisfies (5.5) and (5.6), then there exists a vector $\varphi$ which is cyclic for the representation $\mathfrak{R}_{C}$ and satisfies

$$
U \varphi=\pi\left(m_{0}\right) \varphi .
$$

Proof. Using the decomposition given in Theorem 5.1 we can move the representation to $L^{2}\left(E, l^{2}(\mathbb{Z})\right)$. We then have that $\varphi(x) \in l^{2}(\mathbb{Z})$ and

$$
U_{x_{C}} \varphi(x)=\pi_{x_{C}}\left(m_{0}\right) \varphi(x),
$$

for almost all $x \in E$. This rewrites as

$$
\varphi(x)(k-1)=m_{0}\left(\left(x_{C}\right)_{k}\right) \varphi(x)_{k} \quad(k \in \mathbb{Z}) .
$$

Since $\varphi$ is cyclic for the representation, $\varphi(x) \neq 0$ for almost all $x \in E$ (see Proposition 4.9). So there must be some $k_{x}$ such that $\varphi(x)\left(k_{x}\right) \neq 0$. Iterating (5.7), we obtain that

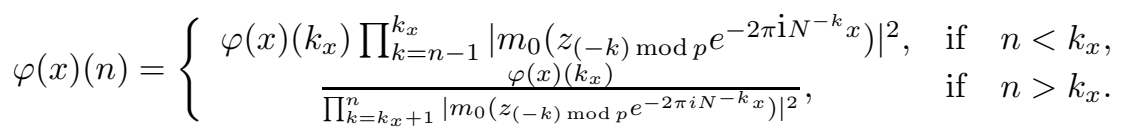

Since $\varphi(x) \in l^{2}(\mathbb{Z})$ for almost all $x$ and $\varphi(x)\left(k_{x}\right) \neq 0$, (5.5) and (5.6) follow.

For the converse, define

$$
\varphi(x)\left(k_{x}\right)=c_{x},
$$


$c_{x}$ being some non-zero constant that we will compute later. Define $\varphi(x)(k)$ by (5.8) for $k \in \mathbb{Z}$. Equation (5.6) implies that there are no zeros in the denominators, so $\varphi(x)(k)$ is well defined, it satisfies (5.7), and, using the hypothesis, $\varphi(x)$ in $l^{2}(\mathbb{Z})$ for almost all $x$. Now take $c_{x}$ such that $\|\varphi(x)\|_{2}=1$. Then we get that $\varphi \in L^{2}\left(E, l^{2}(\mathbb{Z})\right)$.

Also, from (15.7) it follows that $U \varphi=\pi\left(m_{0}\right) \varphi$.

The fact that $\varphi$ is cyclic for the representation follows from Proposition 4.9.

Corollary 5.5. Let $m_{0} \in L^{\infty}(\mathbb{T})$ and let $M:=\left\|m_{0}\right\|_{\infty}$. Suppose the following conditions are satisfied: the following limit exists for almost all $x \in\left(-N^{p},-1\right] \cup$ $\left[1, N^{p}\right)$, and

$$
\lim _{n \rightarrow \infty}\left|m_{0}\left(\frac{x}{N^{k}}+\theta_{-k}\right)\right|>1
$$

There exists $1>\epsilon \geq 0$ such that

$$
\mu\left(\left\{\left.z \in \mathbb{T}|| m_{0}(z)\right|^{2} \leq \epsilon\right\}\right)>\frac{\ln M}{\ln \frac{M}{\epsilon}} .
$$

(When $\epsilon=0$ this means that $m_{0}$ is zero on a set of positive measure.)

Then there exists a scaling vector $\varphi \in L^{2}(\mathbb{R})^{p}$ which is cyclic for the representation $\mathfrak{R}_{C}$ and satisfies the scaling equation

$$
U \varphi=\pi\left(m_{0}\right) \varphi
$$

Proof. We use Theorem 5.4. Using the ratio test, (5.9) implies (5.6).

Let

$$
A_{\epsilon}:=\left\{\left.z \in \mathbb{T}|| m_{0}(z)\right|^{2} \leq \epsilon\right\}, \quad \delta:=\frac{\ln M}{\ln \frac{M}{\epsilon}} .
$$

Since the map $z \mapsto z^{N}$ is ergodic, using Birkhoff's theorem we have that, for almost every $z \in \mathbb{T}$,

$$
\lim _{m \rightarrow \infty} \frac{1}{m} \sum_{k=0}^{m-1} \chi_{A_{\epsilon}}\left(z^{N^{k}}\right)=\mu\left(A_{\epsilon}\right) .
$$

Then, using (5.10), there exists $m_{x}$ and some $\delta^{\prime}>\delta$ such that, for $m \geq m_{x}$,

$$
\frac{1}{m} \sum_{k=0}^{m-1} \chi_{A_{\epsilon}}\left(z^{N^{k}}\right)>\delta^{\prime} .
$$

This can be rewritten as

$$
k_{\epsilon, z}:=\sharp\left\{k \leq m-1 \mid z^{N^{k}} \in A_{\epsilon}\right\}>m \delta^{\prime} .
$$

Therefore we have

$$
\begin{aligned}
\prod_{k=0}^{m-1}\left|m_{0}\left(z^{N^{k}}\right)\right|^{2} & \leq \epsilon^{k_{\epsilon, z}} M^{m-k_{\epsilon, z}} \leq \epsilon^{m \delta^{\prime}} M^{m\left(1-\delta^{\prime}\right)} \\
& =e^{m\left(\delta^{\prime} \ln \epsilon+\left(1-\delta^{\prime}\right) \ln M\right)} .
\end{aligned}
$$

Since $\delta^{\prime}>\delta=\ln M /(\ln (M / \epsilon))$, it follows that $\gamma:=\delta^{\prime} \ln \epsilon+\left(1-\delta^{\prime}\right) \ln M<0$. Therefore

$$
\sum_{m=m_{x}}^{\infty} \prod_{k=0}^{m-1}\left|m_{0}\left(z^{N^{k}}\right)\right|^{2} \leq \sum_{m=m_{x}}^{\infty} e^{m \gamma}<\infty .
$$


Take $z=e^{-2 \pi \mathrm{i}\left(x / N^{l}+\theta_{-l}\right)}$ with $x \in \mathbb{R}$ and $l\left(=k_{x}\right) \in \mathbb{Z}$, and note that $z^{N^{k}}=$ $e^{-2 \pi i\left(x / N^{l-k}+\theta_{-l+k}\right)}$; we get that

$$
\sum_{m=m_{x}}^{\infty}\left|m_{0}\left(\frac{x}{N^{k_{x}}}+\theta_{-k_{x}}\right) m_{0}\left(\frac{x}{N^{k_{x}-1}}+\theta_{-k_{x}+1}\right) \ldots m_{0}\left(\frac{x}{N^{k_{x}-m}}+\theta_{-k_{x}+m}\right)\right|^{2}<\infty .
$$

Neglecting the first few terms, and reversing the order in the products, (5.5) is obtained and the corollary is proved.

We remark that, if $\epsilon=0$, the zero set has positive measure and the ergodicity implies that almost every trajectory must go through the zero set. Therefore the products appearing in (5.5) are zero, so we are summing zero terms.

5.2. Representations on fractals. We saw in DutJo that, if one takes

$$
N=3 \quad \text { and } \quad m_{0}(z)=\frac{1+z^{2}}{\sqrt{2}}
$$

then the representation associated to this filter (as in Theorem 1.1) can be constructed on a Hausdorff measure.

More precisely, consider $\mathcal{R}$, the set of all real numbers that have a base 3 expansion containing only finitely many 1's. On this set take the Hausdorff measure $\mathcal{H}^{s}$ with $s=\log _{3} 2$ - the Hausdorff dimension of the triadic Cantor set. Define the unitary operators

$$
\begin{gathered}
U f(x)=\frac{1}{\sqrt{2}} f\left(\frac{x}{3}\right) \quad\left(x \in \mathcal{R}, f \in L^{2}\left(\mathcal{R}, \mathcal{H}^{s}\right)\right), \\
T f(x)=f(x-1) \quad\left(x \in \mathcal{R}, f \in L^{2}\left(\mathcal{R}, \mathcal{H}^{s}\right)\right),
\end{gathered}
$$

and $\varphi=\chi_{\mathbf{C}}$, where $\mathbf{C}$ is the triadic Cantor set.

We will use the techniques developed in the previous sections to analyze in more detail this representation, give it another form and then to define a possible Fourier transform related to the Hausdorff measure $\mathcal{H}^{s}$.

For our $m_{0}$, the corresponding function $W=\left|m_{0}\right|^{2} / 3$ is

$$
W(z)=\frac{1}{3}+\frac{1}{6} z^{2}+\frac{1}{6} z^{-2},
$$

therefore the coefficients are $a_{0}=\frac{1}{3}, a_{2}=a_{-2}=\frac{1}{6}$ and all others are 0 .

We analyze the measure $m$ on $\mathcal{S}_{N}$ constructed from $W$ as in Theorem 4.3 ,

Proposition 5.6. The measure $m$ has the following Fourier coefficients:

$$
\hat{m}(\lambda)=\left\{\begin{array}{cc}
2^{-\left(\left|d_{0}\right|+\left|d_{1}\right|+\ldots+\left|d_{p}\right|\right) / 2}, & \text { if } \lambda=\sum_{k=0}^{p} \frac{d_{k}}{3^{k}}, \text { with } d_{k} \\
0, & \text { otherwise. }
\end{array}\right.
$$

Proof. Equation (4.7) becomes in our case

$$
\hat{m}(\lambda)=\frac{1}{2} \hat{m}(3 \lambda-2)+\hat{m}(3 \lambda)+\frac{1}{2} \hat{m}(3 \lambda+2) \quad(\lambda \in \mathbb{Z}[1 / 3]) .
$$

We will use another lemma, which is also interesting in its own, because it tells something about the geometry of the Cantor set.

\section{Lemma 5.7.}

$$
\hat{m}(\lambda)=\mathcal{H}^{s}((\mathbf{C}+\lambda) \cap \mathbf{C}) \quad(\lambda \in \mathbb{Z}[1 / 3]) .
$$


Proof of Lemma 5.7. We saw in Theorem 4.3 that the representation associated to $m_{0}$ can be realized on $L^{2}(m)$ with scaling function $\varphi^{\prime}=1$. In this representation, the translation by $\lambda$ is given by multiplication by the character $\chi_{\lambda}$; therefore we have

$$
\hat{m}(\lambda)=\left\langle T_{\lambda}^{\prime} \varphi^{\prime} \mid \varphi^{\prime}\right\rangle .
$$

However, this representation is isomorphic to the one described above, the isomorphism mapping the scaling function $\varphi=\chi_{\mathbf{C}}$ to $\varphi^{\prime}=1$. In this representation, translation by $\lambda$ is simply the translation by $\lambda$ on $\mathcal{R}$. Consequently,

$$
\hat{m}(\lambda)=\left\langle T_{\lambda} \chi_{\mathbf{C}} \mid \chi_{\mathbf{C}}\right\rangle=\mathcal{H}^{s}((\mathbf{C}+\lambda) \cap \mathbf{C}) .
$$

From Lemma 5.7 we deduce that, if $|\lambda| \geq 1$, then $\hat{m}(\lambda)=0$. So, if $\left|\lambda_{1}-\lambda_{2}\right| \geq 2$, then at most one of them is in the interval $(-1,1)$, so at most one of them is non-zero. This implies that in the right side of (5.12), at most one of the terms is non-zero.

Now take $\lambda=l / 3^{n}$. We will proceed by induction on $n$. If $n=0$, the result follows from Proposition 4.2. For $n>0$, assume that $\hat{m}(\lambda)$ is not zero. Using (5.12) and the previous statement, we obtain that exactly one of the terms $\hat{m}(3 \lambda-2), \hat{m}(3 \lambda), \hat{m}(3 \lambda+2)$ is non-zero. Denote by $\alpha=3 \lambda-d_{0}$ the one which is not 0 , with $d_{0} \in\{-2,0,2\}$. Then, analyzing the three cases, we see that

$$
\hat{m}(\lambda)=\frac{1}{2^{\left|d_{0}\right| / 2}} \hat{m}(\alpha) .
$$

But $\alpha$ is of the form $k^{\prime} / 3^{n-1}$, so we can use the induction hypothesis to conclude that $\alpha$ has the form $\alpha=\sum_{l=1}^{p} d_{l} / 3^{l-1}$, so

$$
\lambda=\sum_{l=0}^{p} \frac{d_{l}}{3^{l}}
$$

and (5.11) is proved.

The fact that we have two embodiments of the wavelet representation associated to the filter $m_{0}(z)=\frac{1+z^{2}}{\sqrt{2}}$, one on $\mathcal{R}$ with the Hausdorff measure $\mathcal{H}^{s}$, and the other on $\mathcal{S}_{N}$ with the measure $m$, implies that there is an isomorphism between them which can be interpreted as a Fourier transform on $\mathcal{R}$, since it transforms translations into multiplications.

Corollary 5.8. Consider on $\mathcal{S}_{N}$ the measure $m$ that has Fourier coefficients given in Proposition 5.6. There is a unique isomorphism $\mathcal{F}_{3}$ from $L^{2}\left(\mathcal{R}, \mathcal{H}^{s}\right)$ to $L^{2}\left(\mathcal{S}_{N}, m\right)$ such that for $f \in L^{2}\left(\mathcal{S}_{N}, m\right)$,

$$
\begin{gathered}
\mathcal{F}_{3} T_{\lambda} \mathcal{F}_{3}^{-1} f=\chi_{\lambda} f \quad(\lambda \in \mathbb{Z}[1 / 3]), \\
\mathcal{F}_{3} U \mathcal{F}_{3}^{-1} f=m_{0} f \circ S \\
\mathcal{F}_{3} \chi_{\mathbf{C}}=1
\end{gathered}
$$


5.3. $m_{0}=1$ : Representations on the solenoid. We now take $m_{0}=1$ which obviously satisfies $R_{m_{0}} 1=1$, and we describe the wavelet representation associated to it. The measure $m$ associated to $m_{0}$ as in Proposition 4.2 verifies the scaling equations

$$
\hat{m}(k)=\delta_{k} \quad(k \in \mathbb{Z}), \quad \hat{m}(\lambda)=\hat{m}(N \lambda) .
$$

Therefore

$$
\hat{m}(\lambda)=\delta_{\lambda} \quad(\lambda \in \mathbb{Z}[1 / N]) .
$$

But this means that $m$ is the Haar measure $\mu_{\mathcal{S}_{N}}$ on $\mathcal{S}_{N}$. Hence, from Theorem 4.3 and Example 3.6, we have the following result.

Proposition 5.9. Let $H=L^{2}\left(\mathcal{S}_{N}, \mu_{\mathcal{S}_{N}}\right), T f\left(z_{n}\right)_{n}=z_{0} f\left(z_{n}\right)_{n}, U f=f \circ S(f \in$ $\left.H,\left(z_{n}\right)_{n} \in \mathcal{S}_{N}\right)$. Let $\varphi$ be the constant function 1 on $\mathcal{S}_{N}$. Then $(H, U, T, \varphi)$ is the wavelet representation associated to $m_{0}=1$. The representation is irreducible.

With Corollary 4.6 we have

Corollary 5.10. The only functions $h \in L^{\infty}(\mathbb{T})$ that satisfy

$$
\frac{1}{N} \sum_{k=0}^{N-1} h\left(\frac{\theta+2 k \pi}{N}\right)=h(\theta) \quad(\theta \in[-\pi, \pi))
$$

are the constants.

\section{REFERENCES}

[BDP] S. Bildea, D.E. Dutkay, G. Picioroaga, MRA Super-wavelets, New York J. Math., 11 (2005), 1-19. MR2154344 (2006b:42048)

[Dau92] I. Daubechies, Ten Lectures on Wavelets, CBMS-NSF Regional Conf. Ser. in Appl. Math., vol. 61, Society for Industrial and Applied Mathematics, Philadelphia, 1992. MR.1162107(93e:42045)

[Dut2] D.E. Dutkay, The wavelet Galerkin operator, J. Operator Theory, 51(2004), 49-70. MR2055804 (2005c:47041)

[Dut3] D.E. Dutkay, Positive definite maps, representations and frames, Reviews in Mathematical Physics, vol. 16, No. 4 (2004), 1-27. MR2065233 (2005e:42115)

[DutJo] D.E. Dutkay, P.E.T. Jorgensen, Wavelets on fractals, to appear in Revista Matematica Iberoamericana.

[Fol] G. Folland, A Course in Abstract Harmonic Analysis, CRC Press, 1995. MR:1397028 (98c:43001)

[GS] G. Greschonig, K. Schmidt, Ergodic decomposition of quasi-invariant probability measures, Colloq. Math. 84/85 (2000), part 2, 495-514. MR1784210(2001i:28021)

[Gun00] R.F. Gundy, Low-pass flters, martingales, and multiresolution analyses, Appl. Comput. Harmon. Anal., 9 (2000), 204-219. MR 1777126 (2002k:94013)

[Jor01] P.E.T. Jorgensen, Ruelle operators: Functions which are harmonic with respect to a transfer operator, Mem. Amer. Math. Soc., 152, no. 720. MR1837681 (2002c:46117)

[Jor04] P.E.T. Jorgensen, Processes, wavelets and random walks on branches, preprint.

[LPT] L.-H. Lim, J. Packer, K. Taylor, A direct integral decomposition of the wavelet representation, Proc. Amer. Soc., 129, No. 10, 3057-3067. MR.1840112 (2002c:47146)

[MV00] F. Martin, A. Valette, Markov operators on the solvable Baumslag-Solitar groups, Experimental Mathematics, Vol. 9 (2000), No. 2. MR1780213 (2002a:37005)

[Wal] P. Walters, An introduction to ergodic theory, Springer-Verlag, New York, 1982. MR0648108 (84e:28017)

[Web] E. Weber, Discrete wavelet transforms and $\sigma$-admissible group representations, preliminary version.

Department of Mathematics, Hill Center-Busch Campus, Rutgers, The State UniverSity of New Jersey, 110 Frelinghuysen Road, Piscataway, New Jersey 08854-8019

E-mail address: ddutkay@math.rutgers .edu 